\title{
Editorial
}

\section{Differential Games and Discrete Dynamics in Applied Sciences}

\author{
Massimiliano Ferrara, ${ }^{1,2}$ Gafurjan Ibragimov, ${ }^{3}$ and Vincenzo Scalzo ${ }^{4}$ \\ ${ }^{1}$ Department of Law and Economics, University Mediterranea of Reggio Calabria, Via dei Bianchi 2, Palazzo Zani, \\ 89127 Reggio Calabria, Italy \\ ${ }^{2}$ The Invernizzi Centre for Research in Innovation, Organization, Strategy and Entrepreneurship (ICRIOS), Bocconi University, \\ Via Sarfatti 25, 20136 Milan, Italy \\ ${ }^{3}$ INSPEM and Department of Mathematics, Faculty of Science, Universiti Putra Malaysia, 43400 Serdang, Selangor, Malaysia \\ ${ }^{4}$ Department of Economics and Statistics, University of Naples Federico II, Complesso Monte S. Angelo, Via Cintia, 80126 Naples, Italy
}

Correspondence should be addressed to Massimiliano Ferrara; massimiliano.ferrara@unirc.it

Received 29 December 2016; Accepted 4 January 2017; Published 24 January 2017

Copyright (C) 2017 Massimiliano Ferrara et al. This is an open access article distributed under the Creative Commons Attribution License, which permits unrestricted use, distribution, and reproduction in any medium, provided the original work is properly cited.

In many scientific fields, differential games are problems related to the analysis of conflict and strategical interactions in the model of a dynamical system. In particular, in a pursuit-evasion game, one or more pursuers try to capture one or more evaders that try to avoid capture. Contests of pursuit and evasion are among the most widespread, challenging, and important optimization problems that confront mobile agents. But dynamic, stochastic, continuous-space, continuous-time, or discrete-time discrete-space games are usually difficult to handle. Agents that pursue or evade must maintain complex sensory-motor coordination with respect to both a physical environment and a hostile opponent. Because of its extensive applications, such as searching buildings for intruders, traffic control, military strategy, surgical operation, and industrial management, a lot of research has been developed in various directions during the last three decades, not only for that concerns the specific fields of application. In time of these methods, related mathematical tools and models have been intensively studied focusing on all the aspects concerning "dynamics" in a broad sense. In compliance of this aim, by this special issue, we have been interested in articles that explored various aspects of differentiable games and also in dynamics models. This editorial provides a brief review of some concepts related to the subject of the papers published in this collectanea devoted to the onset of differential games and discrete dynamics with applications to various branches of research. "Dynamics" is currently an active and fashionable discipline that is having a profound effect on a wide variety of fields, including engineering, physics, biology, economics, finance, and social sciences.

By the present work we are going to present some selected papers which analyze various aspects and applications fall in the "dynamics" following a "discrete" mathematical approach.

The paper entitled "Linear Pursuit Differential Game under Phase Constraint on the State of Evader" by M. Ferrara et al. deals with the linear pursuit differential games where pursuit control is subjected to integral constraints and evasion control is subjected to geometric constraint, with phase constraint being imposed on the state of the evader. For such constraints on controls of players, to obtain solution of pursuit problem for all initial states of phase space is complicated because of boundedness of pursuit resources. By this work, the following technique is proposed to control the phase vector of the pursuer: first-phase vector of the pursuer is brought to a given bounded, convex set, regardless of the behavior of evading player and then, using the evasion control and initial positions of the players, the pursuit control that ensures the completion of pursuit is constructed.

In "Simple Motion Pursuit and Evasion Differential Games with Many Pursuers on Manifolds with Euclidean Metric," M. Ferrara et al. consider pursuit and evasion differential games of a group of pursuers and one evader on manifolds with Euclidean metric. The authors establish that each of the differential games (pursuit or evasion) is equivalent to a differential game of groups of countably many pursuers and one group of countably many evaders in Euclidean space. All the players in any of these groups are 
controlled by one controlled parameter. It has been found a condition under which pursuit can be completed, and if this condition is not satisfied, then evasion is possible. Some strategies for the pursuers in pursuit game which ensure completion of the game for a finite time have been given. In the specific case of evasion game, it was given a strategy for the evader.

By the paper entitled "New JLS-Factor Model versus the Standard JLS Model: A Case Study on Chinese Stock Bubbles" Z. Hu and C. Li extend the Johansen-Ledoit-Sornette (JLS) model by introducing fundamental economic factors in China (including the interest rate and deposit reserve rate) and the historical volatilities of targeted and US equity indices into the original model, which is a flexible tool to detect bubbles and predict regime changes in financial markets. A general method has been introduced to incorporate these selected factors in addition to the log-periodic power law signature of herding and compare the prediction accuracy of the critical time between the original and the new JLS models (termed the JLS-factor model) by applying these two models to fit two well-known Chinese stock indices in three bubble periods. The results showed that the JLS-factor model with Chinese characteristics successfully depicts the evolutions of bubbles and "antibubbles" as well as constructs efficient end-of-bubble signals for all bubbles in Chinese stock markets. In addition, the results of standard statistical tests demonstrate the excellent explanatory power of these additive factors and confirm that the new JLS model provides useful improvements over the standard JLS model.

$\mathrm{X}$. Meng et al. in the work entitled "Worst-Case Investment and Reinsurance Optimization for an Insurer under Model Uncertainty" study optimal investment-reinsurance strategies for an insurer who faces model uncertainty. The insurer is allowed to acquire new business and invest into a financial market which consists of one risk-free asset and one risky asset whose price process is modeled by a geometric Brownian motion. Minimizing the expected quadratic distance of the terminal wealth to a given benchmark under the "worst-case" scenario, the authors obtain the closed-form expressions of optimal strategies and the corresponding value function by solving the Hamilton-Jacobi-Bellman (HJB) equation. Numerical examples have been presented to show the impact of model parameters on the optimal strategies.

In "Numerical Approach Based on Two-Dimensional Fractional-Order Legendre Functions for Solving Fractional Differential Equations" a robust numerical approach is proposed to obtain the numerical solution of some fractional differential equations. The principal characteristic of the approach proposed by F. Zhao et al. was the new orthogonal functions based on shifted Legendre polynomials to the fractional calculus. Also the fractional differential operational matrix is driven. Then the matrix with the "Tau method" is utilized to transform this problem into a system of linear algebraic equations. By solving the linear algebraic equations, the numerical solution is obtained. The approach is tested via some examples. It is shown that the FLFs yield better results. Finally, error analysis shows that the algorithm is convergent.

In "Dynamical Analysis of a Computer Virus Model with Delays," J. Liu et al. have proposed an SIQR computer virus model with two delays. The linear stability conditions are obtained by using characteristic root method and the developed asymptotic analysis shows the onset of a Hopf bifurcation occurs when the delay parameter reaches a critical value. Moreover the direction of the Hopf bifurcation and stability of the bifurcating period solutions are investigated by using the normal form theory and the center manifold theorem. Finally, numerical investigations are carried out to show the feasibility of the theoretical results.

By "A Note on Discrete Multitime Recurrences of Samuelson-Hicks Type" B. A. Pansera and F. Strati aim at fostering further research on the applications of multitime recurrences in the frame of multitime theory. In particular, the authors apply this method by generalizing the SamuelsonHicks model so as to make the new concept of time that this method proposes clear. In particular, the multitime approach decomposes a point of time into a vector, taking into account how different coordinates of time referring to the same date can affect the dynamics of a model. Since 1932, the adjective multitime, introduced by Paul Dirac, appears so as to cope with an environment in which there might be more than one dimension of time. The authors make some considerations concerning the physical idea of the multitime notion: they recall that a coordinate space is an index numbering degree of freedom, and the coordinate of time is the usual physical time in which a system evolves. This model is satisfactory, unless the reader turns his attention to relativistic problems. Moreover, some physical phenomena (and social sciences too) are observed in a two-time environment, where the intrinsic time and the observer time are found. In some real phenomena, there is no reason to prefer one coordinate to another. Following these assumptions, the authors refer to multitime as vector parameters of evolution in multitime geometric evolution and multitime optimal control problems.

Constructing a Poincaré map is a method that is often used to study high-dimensional dynamical systems. In "Topological Entropy of One Type of Nonoriented LorenzType Maps," G. Feng has proposed a geometric model of nonoriented Lorenz-type attractor by using this method, and its dynamical property has been described. The topological entropy of one-dimensional nonoriented Lorenz-type maps is also computed in terms of their kneading sequences.

In "Dynamic Complexities in 2-Dimensional DiscreteTime Predator-Prey Systems with Allee Effect in the Prey," L. Ren et al. have presented a study by which the "Allee effect" is incorporated into a predator-prey model with linear functional response. Compared with the predator-prey which only takes the crowding effect and predator partially dependent on prey into consideration, it has been found that the "Allee effect" of the prey species would increase the extinction risk of both the prey and predator. Moreover, by using a center manifold theorem and bifurcation theory, it has been shown that the model with "Allee effect" undergoes the flip bifurcation and Hopf bifurcation in the interior of $R_{+}^{2}$ with different "Allee effect" values. In the two bifurcations, the authors came to the conclusion that different "Allee effects" have different bifurcation value and the increasing of the "Allee effect" increases the value of bifurcation, respectively. 
By "Existence of Nonoscillatory Solutions of HigherOrder Neutral Differential Equations with Distributed Coefficients and Delays," Y. Liu et al. have considered the existence of nonoscillatory solutions of higher-order neutral differential equations with distributed coefficients. The authors have used the contraction principle in obtaining new sufficient condition for the existence of nonoscillatory solutions.

In the paper entitled "Modeling and Nonlinear Response of the Cam-Follower Oblique-Impact System" by Y.-F. Yang et al., in order to quickly and accurately analyze the complex behavior of cam-follower oblique-impact system, a mathematical model, which can describe separation, impact, and contact, was established. The transient impact hypothesis was extended, and the oblique collision model was established by considering the tangential slip. "Moreau time-stepping method" was employed to solve the linear complementarity problem transformed by the oblique-impact equations. The simulation results have been organized showing that the cam and follower kept permanent contact when the cam rotational speed was low. With the increase of the cam rotational speed, the cam and follower would be separated and then have impact under the gravity action. The system performance has shown very complex nonlinear characteristics.

In "Risk-Averse Evolutionary Game Model of Aviation Joint Emergency Response," W. Pan et al. have studied effects of risk-averse attitude of both participators in aviation joint emergency response on the coevolution of cooperation mechanisms and individual preferences between airport and nonprofit organization. First, based on the current aviation joint emergency mechanism in China, the authors put forward two mechanisms to select the joint nonprofit organization, including reputation cooperation and bidding competition. Meanwhile, they have considered two preferences including altruism and selfishness. Then "replicator dynamics equations" by using the theory of conditional value-at-risk (CVaR) taking risk aversion attitude into account were built. Finally, the authors show that the risk-averse attitude of the other game participator affects the one participator's decision and the effects subject to some parameters.

The guest editors of this special issue hope that problems discussed and investigated in the papers by the authors of this issue can inspire and motivate researchers in these fields to discover new, innovative, and novel applications in all areas of pure and applied mathematics.

\title{
Acknowledgments
}

We would like to express our great gratitude to all of the authors for their contributions and the reviewers for their serious evaluation of the papers submitted by valuable comments and timely feedback.

\author{
Massimiliano Ferrara \\ Gafurjan Ibragimov \\ Vincenzo Scalzo
}




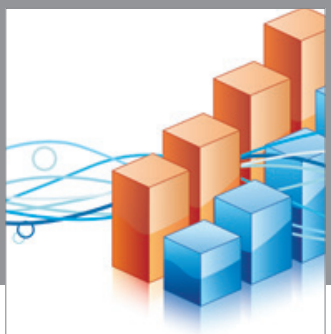

Advances in

Operations Research

vatem alat4

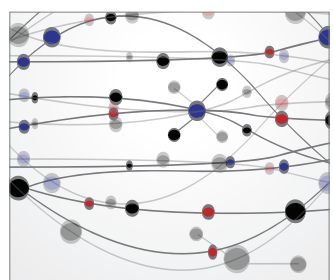

\section{The Scientific} World Journal
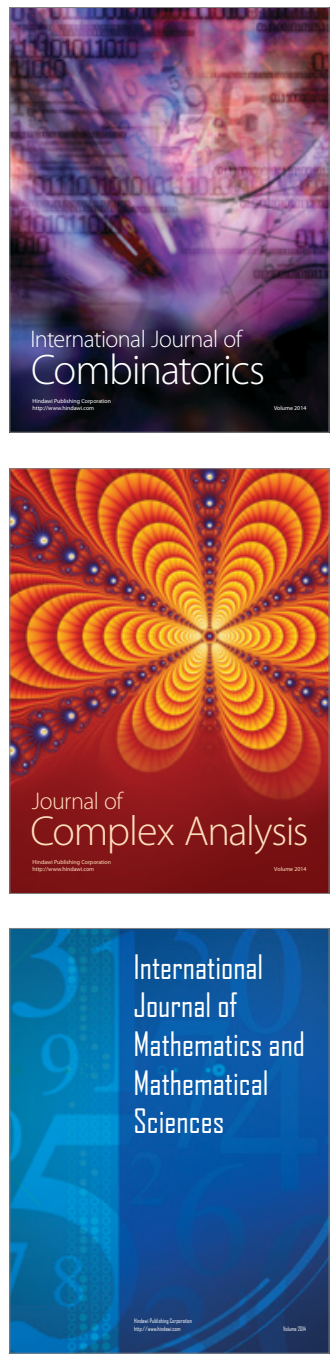
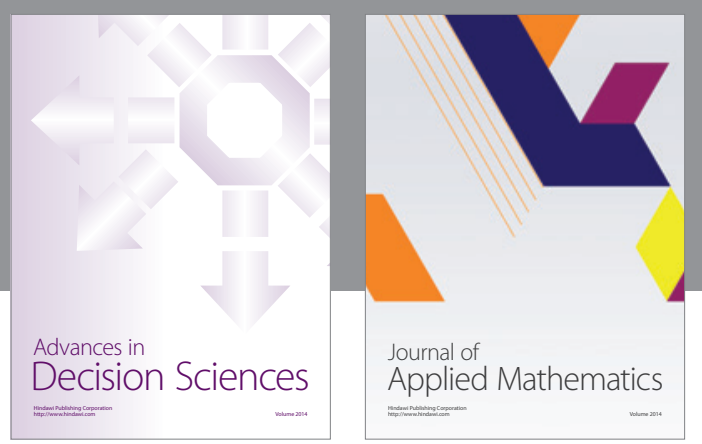

Algebra

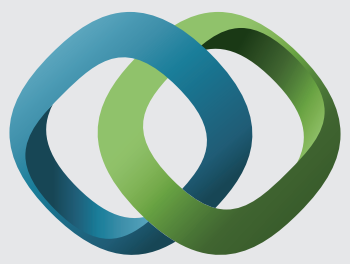

\section{Hindawi}

Submit your manuscripts at

https://www.hindawi.com
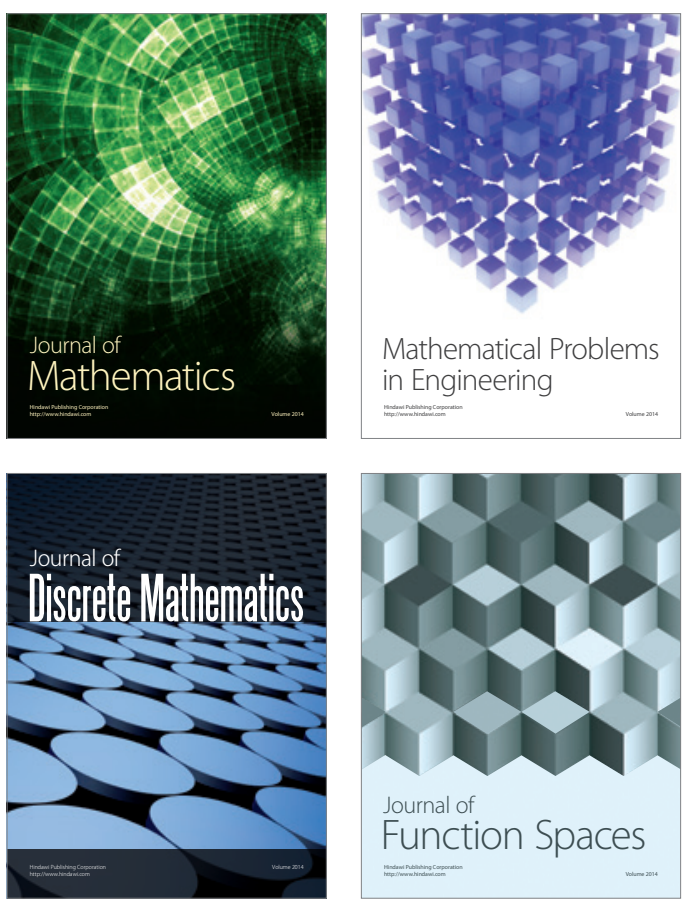

Mathematical Problems in Engineering
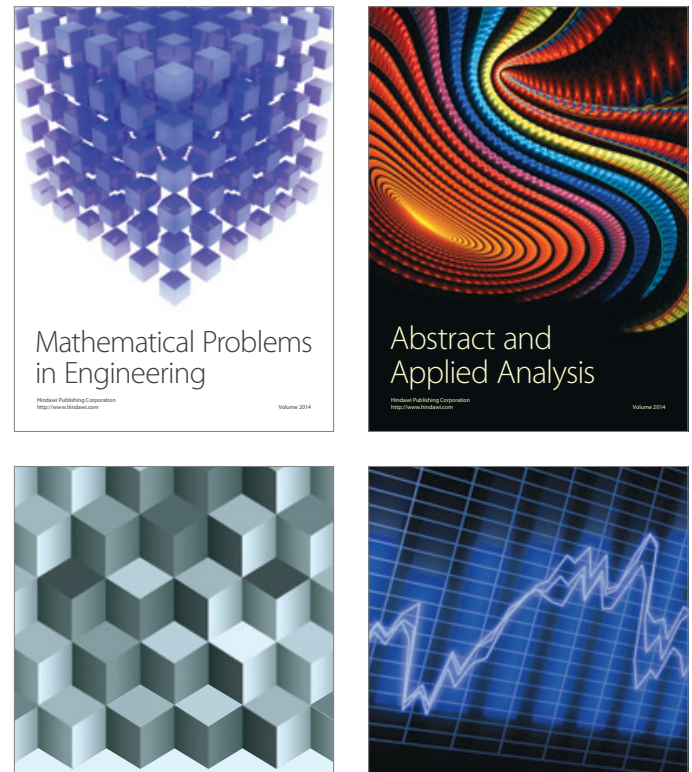

Journal of

Function Spaces

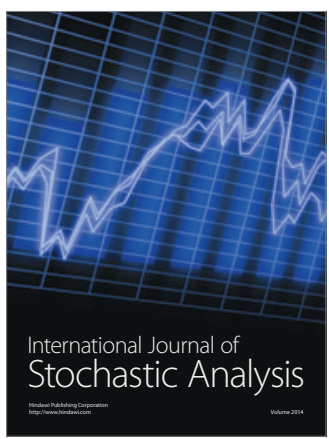

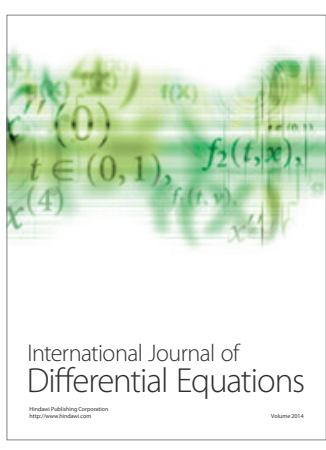
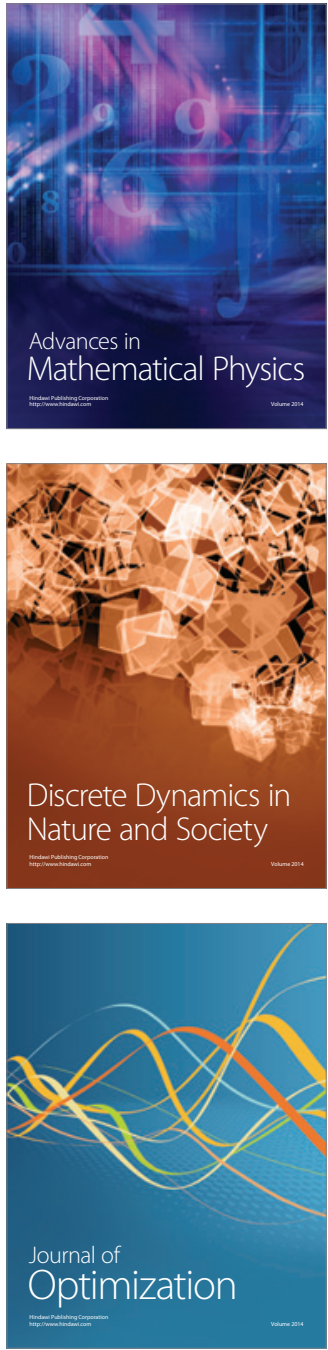